TABLE DES MATIËRES

Rapport Science et Technologie iii

Composition du Comité RST v

Résumé ix

Summary xiii

Préface Xvii

Avant-propos $\quad$ xix

Composition du groupe de travail $\quad$ xxi

Introduction $\quad$ xxxiii

Synthèse $\quad$ xxxvii

Recommandations Iv

Recommendations Ixxi

PREMIÈRE PARTIE Les contraintes 1

CHAPITRE 1 Les évolutions démographiques 3

1. Les contraintes démographiques . . . . . . . . . . . . . . . 5

1.1. Méthodes et valeur des projections démographiques . . . . . 5

1.2. Les projections actuelles et leurs variantes . . . . . . . . . . . 7

1.3. Où les 3 milliards d'habitants supplémentaires vivront-ils? . . . 9

1.4. Le problème des migrations . . . . . . . . . . . . . . 13

2. Risques et conséquences d'évolutions démographiques non prévues . . 17 


\section{CHAPITRE 2 Les impacts potentiels des changements climatiques et des événements extrêmes sur la production agricole et effets réciproques}

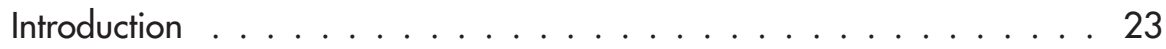

1. Conséquences des changements climatiques . . . . . . . . . . . 24

2. Effet sur la production agricole de l'augmentation de la teneur de l'atmosphère en $\mathrm{CO}_{2}$ couplée à l'augmentation de la température . . . . . . . . . . . . . . . . 25

3. Effet potentiel en retour de l'augmentation de la production agricole sur le climat . . . . . . . . . . . . . . . . . . 27

4. Évolutions climatiques imprévues ou extrêmes . . . . . . . . . . 36

Conclusion . . . . . . . . . . . . . . . . . . . . . . . . . 41

CHAPITRE 3 La demande alimentaire actuelle : facteurs d'évolution

1. Situation alimentaire actuelle et inégalités . . . . . . . . . . 47

1.1. Sous-alimentation chronique en énergie et disponibilités alimentaires . . . . . . . . . . . . . . . . . . 47

1.2. Malnutritions maternelle et infantile . . . . . . . . . 53

1.3. Carences en micronutriments . . . . . . . . . . . . . . . 57

1.4. Impact de la hausse des prix alimentaires et de la crise économique mondiale sur les malnutritions . . . . . . . . . 61

1.5. Changements dans les régimes alimentaires et obésité . . . . 63

1.6. Proposition . . . . . . . . . . . . . . 67

2. Évolution des besoins 2000-2050 . . . . . . . . . . . . . 67

2.1. Alimentation, environnement, changement climatique . . . . . 68

2.2. Quelles stratégies pour une alimentation durable en 2050 ? . . . 71

\section{CHAPITRE 4 Biodiversité, agriculture et environnement 79}

1. Qu'est-ce que la biodiversité? . . . . . . . . . . . . . . . . . 81

2. Comment concilier la préservation de la biodiversité avec l'augmentation nécessaire de la production agricole?

2.1. Séparer les espaces d'agriculture intensive et de protection de la nature

2.2. Promouvoir une agriculture respectueuse de l'environnement : une logique de fonctionnement écologique à l'échelle des territoires 
2.3. Évolution des politiques de protection : espace et biodiversité

2.4. Éviter la perte des surfaces agricoles : le problème des petits exploitants

\section{DEUXIÈME PARTIE Les moyens de la production alimentaire en 2050}

CHAPITRE 5 Moyens techniques et ressources

Introduction

1. Disponibilité en terres

1.1. Les chiffres bruts à l'échelle de la planète . . . . . . . . . . . 102

1.2. Les situations par continent . . . . . . . . . . . . . . 103

1.3. Les pertes en terres agricoles . . . . . . . . . . . . . 105

2. Nouveaux modes de production . . . . . . . . . . . . . . . 107

3. Limitations physiques et biologiques à la production végétale Le rôle de l'amélioration des plantes . . . . . . . . . . . . . . . 117

3.1. Les contraintes de la production . . . . . . . . . . . . 117

3.2. Mieux utiliser les ressources . . . . . . . . . . . . . . . 119

3.3. Mieux résister aux agresseurs . . . . . . . . . . . . . 124

Conclusion . . . . . . . . . . . . . . . . . . . . . . 125

4. Élevage : perspectives et limites au développement des productions animales . . . . . . . . . . . . . . . . . . . 127

5. Ressources halieutiques . . . . . . . . . . . . . . . . . . . 131

5.1. La pêche fournit aux hommes un apport protéique essentiel, pour combien de temps encore?

5.2. L'aquaculture : évolution des productions et enjeux de recherche 133

6. Réduction des pertes . . . . . . . . . . . . . . . 138

6.1. Pertes et gaspillages : état des connaissances . . . . . . . . 139

6.2. Étape récolte et post-récolte . . . . . . . . . . . . . . 140

6.3. Étape transport et commerce de gros . . . . . . . . . . . . . 141

6.4. Étape commerce de détail et consommation . . . . . . . . . . 141

6.5. Synthèse et impacts des pertes . . . . . . . . . . . . . . 148

6.6. Réduire les pertes? . . . . . . . . . . . . . . . . 150

7. Marges de manœuvre concernant les ressources . . . . . . . . . 151

8. Utilisations non alimentaires de la biomasse et concurrence pour l'utilisation des terres : belles promesses ou vraies craintes? . . . 152

8.1. Définitions et ordres de grandeur . . . . . . . . . . . . 153 
8.2. Le développement des biocarburants de première génération et leur responsabilité dans la flambée 2006-2008 des cours agricoles

8.3. Perspectives de développement des utilisations non alimentaires de la biomasse . . . . . . . . . . . . . . . . . 157

\section{CHAPITRE 6 L'environnement économique}

1. Conditions économiques

1.1. Les tendances longues des prix sur les marchés alimentaires internationaux

1.2. La question de la sécurité alimentaire . . . . . . . . . 176

2. Causes économiques de déséquilibres, pauvreté . . . . . . . . . . . 179

2.1. Conséquences de crises économiques majeures . . . . . . . . . 179

2.2. La volatilité des prix . . . . . . . . . . . . . . . . . . . . 184

2.3. La question de la régulation des marchés internationaux . . . 188

2.4. L'articulation entre marchés internationaux et marchés intérieurs 190

3. L'assurance contre les risques climatiques - Perspectives pour les pays en développement . . . . . . . . . . . . . . . . . . . . . 191

3.1. Assurances fondées sur des index météorologiques . . . . . . . 191

3.2. Obligations catastrophes (Cat Bonds) . . . . . . . . . . . 192

3.3. Discussion . . . . . . . . . . . . . . . . . . . . . . . 192

CHAPITRE 7 Les scénarios d'équilibre entre population, alimentation et climat 195

1. Les divers scénarios utilisés . . . . . . . . . . . . . . . . . . 197

2. Comparaison des scénarios. Synthèse . . . . . . . . . . . . 198

3. La question des calories d'origine animale . . . . . . . . . . . 200

Conclusion générale

Annexes

ANNEXE 1 Présentation des scénarios du chapitre 7

Les scénarios d'équilibre entre population, alimentation et climat 
Groupe de lecture critique

Composition du Groupe de lecture critique . . . . . . . . . . . . . . 253

Commentaire de l'Association «Équilibres et populations » . . . . . . 255

Commentaire de la Conférence des grandes écoles . . . . . . . 257

Commentaire de la Fondation Ensemble . . . . . . . . . . . . 265

Commentaire du GRET (Groupe de recherche et d'échanges technologiques, association de solidarité et de coopération internationale) . . . . . . . . . . . . . . . . . . . 269

Commentaire du Groupe Limagrain . . . . . . . . . . . . . . 275

Commentaire de l'Institut de recherche pour le développement . . . . 283

Commentaire du Ministère des Affaires étrangères et européennes . . 285

Commentaire du Ministère de l'Alimentation, de l'Agriculture et de la Pêche . . . . . . . . . . . . . . . . . . . . . 291

À propos des commentaires reçus sur les aspects économiques du rapport . . . . . . . . . . . . . . . . . . . . . . . . . 295

Présentation à l'Académie des sciences, par François Gros 299 
7 KIVSDJHIQQMQWRQDQIOIKEOQN 